\title{
A SIMULATION MODEL TO JUSTIFY REMANUFACTURING POLICIES
}

\author{
Farhad Azadivar \\ Department of Mechanical Engineering \\ School of Engineering \\ University of Massachusetts Dartmouth \\ Dartmouth, MA02747, USA
}

\author{
Sharon Ordoobadi \\ Department of Decision and Information Sciences \\ Charlton School of Business \\ University of Massachusetts Dartmouth \\ Dartmouth, MA, 02747, USA
}

\begin{abstract}
To maintain a high level of service Original Equipment Manufacturers (OEMs) provide more generous return policies for their products. However, discarding returned parts is neither economical nor environmentally desirable. As a result there is a great need for policies on remanufacturing aftermarket products from returned units. Two of the factors that affect justification of setting up after-market reproduction runs are the flow rate and composition of good returned parts. These factors are stochastic functions of the production rate for the primary product and the reliability of individual subassemblies. A closed form solution for estimating these variables is often difficult to develop. This paper presents a general formulation for simulation modeling of such remanufacturing systems and estimation of the flow rate and composition of good returned parts.
\end{abstract}

\section{INTRODUCTION}

In a competitive market a liberal warranty and return policy provides major advantages for marketing a product. Returned products, however, cause challenges for manufacturers in terms of loss of material and disposal complications. A failed product may in fact contain many non-defective parts that could easily be used in producing after-market units. Furthermore, as more laws are enforced for protecting the environment, disposing of failed units becomes more problematic. An obvious solution for dealing with this problem is to produce aftermarket products using good parts of returned products.

Producing products from recovered good parts may be justified only if, considering all cost elements, aftermarket products cost less than primary products (products assembled from new components in a regular production run). Some of the factors that contribute to lower cost of aftermarket products are:

- Availability of returned parts and subassemblies at little or no additional cost.

- Savings in disposal expenses. These savings tend to be more significant as more environmental protection laws are enforced.

Some of the contributors to the cost of aftermarket products include:

- Cost of disassembly of returned products and sorting of the parts.

- Increase in reassembly costs due to smaller batch sizes and the need to use alternative manufacturing (assembling) processes compared to those used in manufacturing of primary products.

- Inventory holding costs for recovered parts, until a reasonable number of complete kits are accumulated to make remanufacturing feasible.

- After-market product discount that has to be included in the selling price. 


\section{Azadivar, Ordoobadi}

Considering the above factors, the appropriate decision on whether to attempt recycling requires a tool to provide a reasonable estimate of the cost of a remanufactured product for comparison to that of the primary one. However, due to an uncertain supply chain for returned parts estimating the cost of an aftermarket product is a rather complicated stochastic function of many parameters. In particular, this cost depends highly on the lot sizes of kits of good returned parts that could be assembled into complete units.

This paper utilizes computer simulation to estimate the lot size of kits formed of good returned parts for a planning period. This is needed to estimate unit reassembling cost. The simulation model also estimates the inventory levels for returned parts, which is another critical factor contributing to aftermarket product costs. Using these estimates and other prevailing parameters of the system, a decision rule is developed to judge the feasibility of recycling returned parts.

The rest of the paper is structured as follows. Section 2 presents a brief background on the subject. In Section 3 the system parameters are defined, the problem is formulated, and the proposed simulation approach is described. Application of the developed methodology to a typical system is covered in section 4 through a numerical example. Section 5 presents conclusions and remarks as well as a brief description of other dimensions of the problem that are being developed in the ongoing research.

\section{BACKGROUND AND RESEARCH MOTIVATION}

Manufacturers choose to engage in reverse logistics and particularly product recovery to reduce production costs, meet customer demands, enhance their brand image, and protect after-market demands (Toffel 2004; Guide and Van Wassenhove 2002) The focus of the present research is on the remanufacturing function associated with the reverse supply chain of parts resulting from returned products. It is a known fact that many companies design their products with an eye on remanufacturing capabilities (Ferrer and Swaminathan 2006). Thus, here we offer a review of the decision models developed by researchers in the field of reverse supply chain with special focus on remanufacturing function.

Toktay, Wein and Zenios (2000) address the challenges of inventory management in remanufacturing due to uncertainty of product returns. These researchers propose a queuing model to solve the management of product returns in a remanufacturing environment. An inventory control method is proposed by Teunter (2001) for production systems with reverse logistics. The simulation results indicate that the model leads to discounted cash flow (DCF) optimal inventory strategies. An analytical approach is presented by Jayaraman (2006) to address the production planning and control for closed-loop supply chains with product recovery. Teunter, Bayindir and Van Den Heuvel (2006) address the dynamic lot sizing problem for systems with product returns. The overall economics of the disassembly processes, and in particular the cost to disassemble is the focus of the research conducted by Das, Yedlarajiah, and Narendra (2000). Akçli \& Bayindir (2008) analyze the impact of inventory holding cost of the recovered parts in a disassembly and recovery environment.

Ferguson \& Toktay (2006) analyze the competition between new and remanufactured products and identify conditions under which the firm chooses not to remanufacture its products. The study of a firm that produces new products in the first period and uses returned cores to offer remanufactured products along with new products in future periods is conducted by Ferrer \& Swaminathan (2006). A bi-level optimization model is developed by Srivastava (2008) to determine the profitability of value recovery (VR) activities for three product categories. The result of the study shows that it is beneficial for manufacturing companies to implement remanufacturing and refurbishing operations to product returns.

Although remanufacturing of products from recycled parts makes good intuitive sense in improving productivity and protection of the environment, the cited literature do not provide enough specific quantitative tools to assist economic justification of such activities. This paper presents a simulation based tool for making decisions on remanufacturing of products from good parts recovered from returned products by incorporating all the original production parameters. 


\section{PROBLEM FORMULATION}

To justify remanufacturing of aftermarket products this paper assumes that aftermarket products are sold at a price below that of the primary product and therefore each unit should cost less to produce. Two characteristics of the remanufacturing operation that have an important impact on remanufactured product cost are the lot sizes of kits formed from returned parts and their corresponding inventory levels. The assumption made here is that the returned parts are available at no cost. The actual cost items are considered to be those of disassembly and assembly of parts and the inventory carrying cost for holding returned parts until they form an appropriate lot size for a remanufacturing run. Lot sizes affect the reassembly cost because larger lots usually benefit from economy of scale. Inventory levels, on the other hand, affect the inventory carrying. Closed form solutions for estimating lot sizes and inventory levels are not readily available and simulation is perhaps the most efficient method for their evaluation. The way these variables are estimated and used in cost estimation is described below.

The problem is formulated in a dimensionless environment. It is assumed that the production cost of a unit of primary product is 1 and the contribution of costs of subassemblies and parts used in the product as well as the cost of the assembly process are all stated in terms of fractions of the unit primary product cost. Also, even though remanufacturing may consist of some tasks other than assembly, the term "reassembly" is used to indicate all of the processes that are used to build a complete unit out of the recovered good parts/sub-assemblies. Furthermore, for simplicity and without loss of generality, the term "part" is used to refer to both parts and sub-assemblies. The cost of all processes involved in putting together an aftermarket product out of good returned parts is referred to as the reassembly cost. This cost excludes costs of individual parts and subassemblies. With these assumptions, components of unit costs of primary and aftermarket products are derived. This formulation is then used to compare the costs for primary and remanufactured products for any given primary production rate.

\section{ESTIMATION OF COSTS}

\subsection{Primary product cost}

The cost for a unit of primary product is expressed as:

$$
C_{\text {PrimaryProduct }}=C_{\text {PrimaryAssembly }}+\sum_{1}^{K} C_{\text {Part }-k}=1.0
$$

Where,

$C_{\text {PrimaryProduct }}$ : Unit Primary Product Cost

$\mathrm{K}$ : Number of parts in the product

$C_{\text {Part_k }} ; k=1, K:$ Cost of part $\mathrm{k}$ as a fraction of unit primary product cost

$\mathrm{C}_{\text {PrimaryAssembly: }}$ Primary Product Assembly Cost as a fraction of unit primary product cost. This cost includes the cost of all processes employed to build a unit of product excluding the cost of individual parts and subassemblies.

\subsection{Remanufactured product cost}

The cost structure for remanufactured products is different from the above. Without loss of generality the cost allocation option proposed by Toktay \& Wei (2006), where recycled parts are available at no cost, has been adopted. However, even with free parts the cost of aftermarket products may still be higher than the cost of primary products due to higher reassembly process cost, cost of disassembling and sorting of 
parts from returned products, and inventory holding costs of recycled parts. Furthermore, since these products will be sold at after-market prices, a discount cost should be added to each unit. However, in cases where disposal costs are significant, the cost of disposal is considered as a negative expense (because recycling helps in saving these costs) and subtracted from the cost. Then the cost of a unit of remanufactured product, again in terms of fractions of the unit primary product cost, can be stated as:

$$
C_{\text {Aftermarket }}=C_{\text {Reassembly }}+C_{\text {InventoryCarrying }}+C_{\text {Disassemble }}+C_{\text {Discount }}
$$

Where

$C_{\text {Aftermarket }}$ : Unit Aftermarket Product Cost



$C_{\text {InventoryCarrying: }}$ Unit Inventory Carrying Cost allocated to each aftermarket product

$C_{\text {Disassemble: }} \quad$ Unit Disassembly and sorting Cost allocated to each aftermarket product

$C_{\text {Discount }}$ : Unit Discount Cost

A short description of these terms is given below.

\subsubsection{Unit reassembly cost $\left(\mathrm{C}_{\text {Reassembly }}\right)$}

It is assumed that all failed products are returned and included in the collection of good parts for reassembly. If the rate of return is affected by the ease of refund or replacement the effect could easily be accounted for by multiplying the return rate by a fraction proportional to the rate of return. It is also assumed that good parts recovered from returned products are sorted and placed in kits for each unit of aftermarket product. These kits are accumulated for a fixed period of time, part accumulation period, to form a reasonable lot size for starting a remanufacturing run as well as to accommodate regular scheduling plans. The reassembly cost for each recycled kit is assumed to be a decreasing function of the batch size of the accumulated kits. (As stated above the lot sizes of these bacthes are clearly a stochastic function of the primary production rate and the reliability of each part/subassembly type.) These lot sizes are also functions of the accumulation period as well as the way parts match to form a kit. Thus the function to estimate these lot sizes can be stated as:

$$
L_{\text {Remanufacture }}=g\left(R_{\text {PrimaryProduct }}, P_{\text {FailureRate }}, T_{\text {Accumulation }}\right)
$$

Where

$L_{\text {Remanufacture: }} \quad$ Remanufacturing production lot size

$R_{\text {PrimaryProduct }}$ : Primary production rate

$P_{\text {FailureRate }}=\left(P_{\text {FailureRate_k }}, k=1, K\right): \quad$ A vector of failure rate of parts

$T_{\text {Accumulation: }} \quad$ Duration of time the returned parts are accumulated

Above relationship does not explicitly address the matching mechanism for parts to form kits; this is a complicated stochastic process and reinforces the need for simulation modeling. An interesting aspect of this phenomenon is that even if all parts have the same reliability, even in the long run, the difference between inventory levels of individual parts will be unbounded. This means not all good returned parts will find enough matches to form a complete kit.

The decreasing function relating the unit reassembly cost to the remanufacturing production lot size, $C_{\text {Reassembly }}=\boldsymbol{r}\left(L_{\text {Remanufacture }}\right)$, can take many forms depending on the processes used for remanufacturing, but in general it will eventually be stabilized at a value beyond which the cost does not change. Also it is reasonable to assume that its minimum value, in general, will be greater than the assembly cost for the 


\section{Azadivar, Ordoobadi}

primary product. The form assumed here is a decreasing hyperbolical function stabilizing beyond a certain value for the lot size. Once again to preserve the dimensionless nature of the problem the reassembly cost is stated as a fraction, $M_{\text {Reassembly_l }}$, of the assembly cost of the primary product. That is:

$$
C_{\text {Reassembly }}=M_{\text {Reassembly_l }} * C_{\text {PrimaryAssembly }}
$$

where $M_{\text {Reassembly_l }}$ is the unit reassembly cost multiplier for lot size $l=L_{\text {Remanufacture }}$ and $C_{\text {Reassembly }}$ and $C_{P r i-}$ maryAssembly are unit assembly costs for the remanufactured and the primary products as defined above.

\subsubsection{Cost multiplier for reassembly of recycled lot sizes}

A typical hyperbolically decreasing multiplier for the assembly cost is shown in Figure 1 with parameters defined as follows:

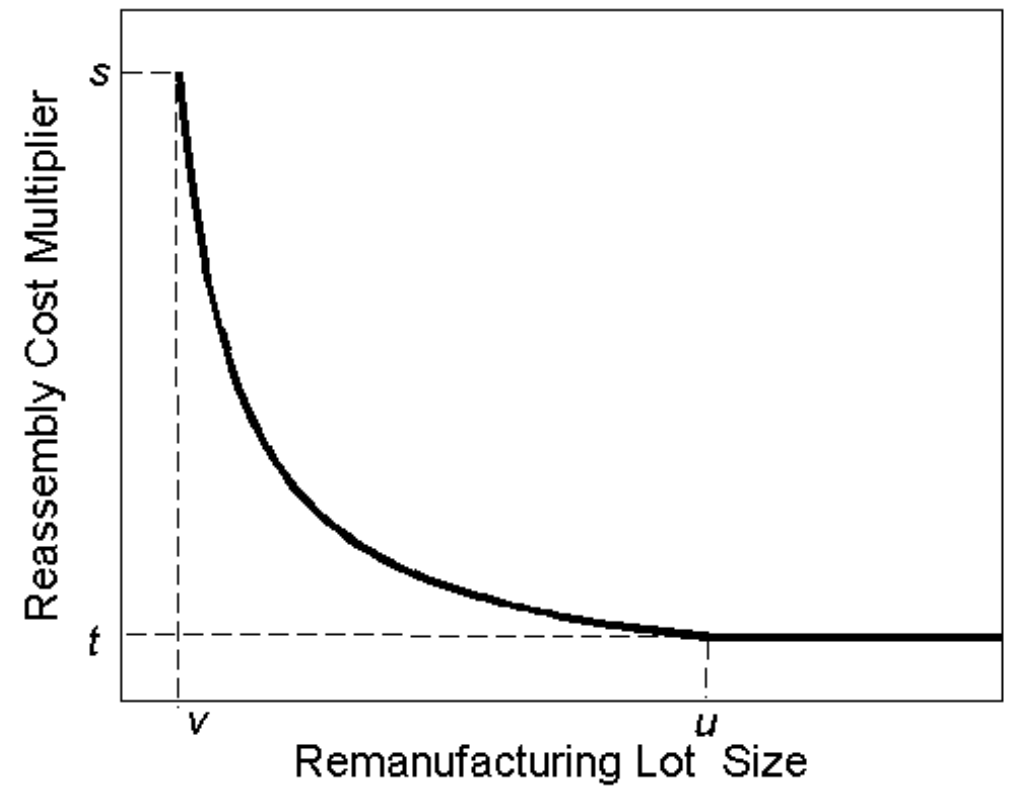

Figure 1: Hyperbolic decreasing reassembly cost model

$z=M_{\text {Reassembly_l }}$

$v$ : absolute minimum batch size to consider remanufacturing;

$s$ : Multiplier for reassembly cost for each product with a lot size $v$;

$u$ : batch size beyond which unit reassembly costs level off;

$t$ : Multiplier for assembly cost for each product in lot sizes greater than $u$.

Values of $s, t, u$, and $v$ depend on the nature of production process and should be considered as the input to the system.

Using above parameters the multiplier function will have the general form of:

$$
z=\frac{a}{l}+b
$$

within values of $u$ and $v$ and of a straight horizontal line for larger values than $u$. Parameters $a$ and $b$ can be estimated from the values of $s, t, u$, and $v$ as follows.

$$
\begin{aligned}
& z=s \text { for } l=v \\
& z=t \text { for } l=u
\end{aligned}
$$




\section{Azadivar, Ordoobadi}

$$
a=\frac{w v(s-t)}{u-v} \quad \text { and } b=\frac{t u-s v}{u-v}
$$

This cost multiplier will be a good approximation if reassembly cost per unit is assumed to consist of a one-time cost due to the set-up plus a fixed cost per unit.

\subsubsection{Unit inventory carrying cost $\left(\mathrm{C}_{\text {InventoryCarrying }}\right)$}

Inventory carrying costs for good parts allocated to each remanufactured product are estimated in the simulation model as follows:

1. Set a planning period.

2. Within this period estimate the holding time for each part before it is reassembled.

3. Multiply the holding time of each part by its corresponding inventory carrying cost per unit time.

4. Add all these costs and then divide the results by the total number of remanufactured products produced during the planning period.

\subsubsection{Unit disassembly and sorting cost $\left(C_{\text {Disassemble }}\right)$}

Disassembly and sorting costs apply to all returned products even though the number of remanufactured products is smaller than the number of returned products (due to discarding of bad parts and not using all good parts). Thus the contribution of these costs to the unit cost of each remanufactured product are estimated by adding the costs for disassembling and sorting of all returned products and averaging the total over the number of remanufactured units.

\subsubsection{Unit disposal costs $\left(\mathrm{C}_{\text {Disposal }}\right)$}

If the disposal cost of each returned product is $C_{\text {Disposal }}$ each remanufactured product causes a savings equal to this amount. As a result this cost should be deducted from the cost of an aftermarket product since it represents the revenue resulted from not paying for disposal. This justifies the consideration of a negative disposal cost for each unit of aftermarket product.

\section{APPLICATIONS}

Using simulation to estimate all cost figures and eventually the total cost of an aftermarket product provides an efficient tool for making strategic decisions on recycling of returned parts. Obviously for a given primary production rate and a set of cost parameters specified above one can determine if building aftermarket products is feasible. Since in this formulation all cost items have been defined in terms of unit cost of the primary product, remanufacturing is justified if the estimated cost is less than 1. A more useful application will be to use this modeling approach to determine the breakeven point for the primary production rate beyond which recycling is profitable. Following numerical example will clarify this utility of the proposed simulation approach.

\section{AN ILLUSTRATIVE EXAMPLE}

The primary product being considered consists of an assembly of 5 parts (subassemblies) each with a $1 \%$ chance of failing during the warranty period ( $99 \%$ reliability). Additional parameters of the system stated as fractions of the unit cost of the primary product are as follows: 
Primary product cost $=1.00$

Assembly cost per unit of primary product $=0.20$

Total cost of all parts in a unit of primary product $=0.80$

Discount for remanufactured products sold as after-market units $=0.20$

Inventory carrying cost per unit per period $=0.0001$

Disassembly and sorting costs for each returned product $=0.1$

$v$ : Minimum remanufacturing lot size $=10$

$u$ : Lot size beyond which reassembly cost does not change $=100$

$s$ : Reassembly cost multiplier for a lot size of $10=6.5$

$t$ : Reassembly cost multiplier for a lot size of $100=2.0$

Parameters for the hyperbolic cost function can be estimated using equation 6 as:

$$
a=\frac{(100)(10)(6.5-20)}{100-10}=50 \text { and } b=\frac{(2)(100)-(65)(10)}{100-2}=1.50
$$

and the unit reassembly cost multiplier is determined as:

$$
\text { Unit Reassembly Cost Multiplier }=\left\{\begin{array}{cc}
z=\frac{50}{l}+1.5 & \text { for } 10 \leq l \leq 100 \\
2 & \text { for } l \geq 100
\end{array}\right.
$$

A computer simulation model was built using ARENA and 20 replications were made for various values of primary production rates for a period of one year. A year is considered as 50 weeks of 40 hours each. Production rates are stated in terms of the number of products per week. Figure 2 shows the average cost of aftermarket product as a function of primary production rates. An example for how the numbers for this figure are calculated is as follows:

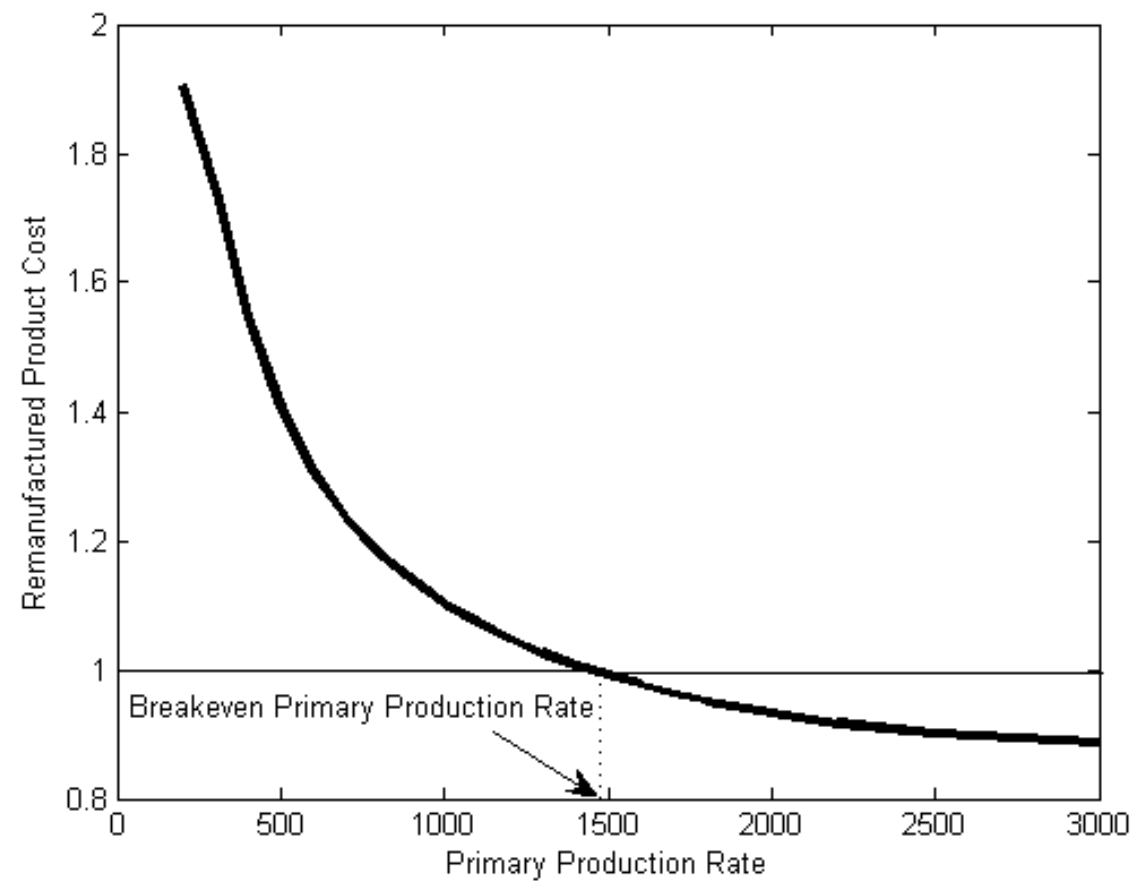

Figure 2: Remanufactured Units Costs as a Function of Primary Production Rate 
For a production rate of 1000 the simulation model provides the following results:

Recycle lot size $=37.627$, Unit sorting cost $=0.129$, Rework holding cost $=0.252$.

From these numbers reassembly cost multiplier can be estimated as $z=\frac{50}{37.63}+1.5=2.828$ resulting a Reassembly cost of $2.828 * 0.2=0.565$. Adding all these costs plus the discount cost of 0.2 results in a total cost for a unit of remanufactured product as 1.102 times the primary product cost.

As indicated in the figure the breakeven point for primary production rate is close to 1500 units per week. If the primary production rate is higher than this breakeven point, remanufactured products' cost will be less than those of the primary products justifying the remanufacturing decision. For lower production rates, remanufactured products will cost more than the primary products, obviously discouraging the remanufacturing decision.

\section{CONCLUSIONS AND SUGGESTIONS FOR FUTURE RESEARCH}

This study presented a quantitative approach for making decisions on whether to recycle the good parts recovered from returned products into remanufactured products that could be sold at after-market prices. It addressed the complicated issue of estimating the flow of good parts recovered from returned products to form reasonable batch sizes for remanufacturing production runs. In particular it demonstrated how a simulation model can be used to estimate very complicated parameters that affect justification of a remanufacturing policy.

The proposed method was developed using a number of relatively reasonable assumptions for the production and cost parameters. These assumptions included fixing a specific time period for accumulation of good parts to form a reasonable number of complete kits. Also, even though the formulation was presented in general terms, the long range accumulation of good parts that do not find their matches, especially when the reliabilities are different for different parts, was not considered. As a matter of fact the preliminary research has indicated that even with the same values of reliability for all parts, still the difference among quantities of different parts may not be bounded. In addition there are also opportunities for building simulation models for cases where bad parts are replaced by new parts to complete kits for recycled products. Clearly adding new parts will increase the material cost but may reduce or even eliminate inventory carrying costs. These and other expansions of the problem are being studied and will be presented in future publications.

\section{REFERENCES}

Akçali, E., and P.Z. Bayindir. 2008. Analyzing the effects of inventory cost setting rules in a disassembly and recovery environment. International Journal of Production Research, 46(1), 267-288.

Das, S., P. Yedlarajiah, and R. Narendra. 2000. An approach for estimating the end-of-life product disassembly effort and cost. International Journal of Production Research, 38(3), 657-673.

Ferrer, G., and J.M. Swaminathan. 2006. Managing new and remanufactured products. Management Science, 52(1), 15-26.

Ferguson, M., and L.B. Toktay. 2006. The effect of competition on recovery strategies. Production and operations management, 46(3), 351-368

Guide, D.V.R., and L.N. Van Wassenhove. 2002. The Reverse Supply Chain. Harvard Business Review, February, 24-24.

Jayaraman, V. 2006. Production planning for closed-loop supply chains with product recovery and reuse: an analytical approach. International Journal of Production Research, 44(5), 981-998.

Srivastava, S. 2008. Value recovery network design for product returns. International Journal of Physical Distribution \& Logistics Management, 38(4), 311-331.

Teunter, R. H. 2001. A reverse logistics valuation method for inventory control. International Journal of Production Research, 39(9), 2023-2035. 
Teunter, R.H., Z.P. Bayindir, and W. Van Den Heuvel. 2006. Dynamic lot sizing with product returns and remanufactutring. International Journal of Production Research, 44(20), 4377-4400.

Toffel, M.W. 2004. Strategic management of product recovery. California Management Review, 46(2), 120-141

Toktay, L.B., L.M. Wein, and S.A. Zenios. 2000. Inventory Management of Remanufacturable Products. Management science 46(11), 1412-1426.

Toktay, L.B., and D. Wei. 2006. Cost allocation in manufacturing - remanufacturing operations. Working paper, Georgia Institute of Technology.

\section{AUTHOR BIOGRAPHIES}

FARHAD AZADIVAR is a professor in the Department of Mechanical Engineering at University of Massachusetts Dartmouth. Prior to that he has served as the dean of College of Engineering at UMASS Dartmouth and the director of Advanced Manufacturing Institute at Kansas State University. His research interests include discrete systems simulation, simulation optimization and the application of simulation based optimization to manufacturing, robotics, transportation, and fishery systems. His e-mail address is <fazadivareumassd.edu>.

SHARON ORDOOBADI is an associate professor in the Department of Decision and Information Sciences in the Charlton College of Business at University of Massachusetts, Dartmouth. Her research interests and publications are in the general area of decision modeling. In particular, her works include application of decision modeling to reverse supply chain, management of technological innovation, and supply chain management. Her e-mail address is <sordoobadi@umassd. edu $>$. 